\title{
Algunos objetivos operativos para la investigación y la educación en Documentación
}

\author{
Francisco Javier García Marco \\ Universidad de Zaragoza (España)
}

\subsection{Resumen}

Se analizan algunos de los retos actuales en la gestión de sistemas de información a partir de la síntesis y discusión de los estudios publicados en el número uno del volumen 10 de la revista Scire (en.-jun. 2004). Los artículos abordan temas novedosos relacionados con la historia de la Documentación, las bases teóricas del análisis de contenido, la construcción de lenguajes documentales en el campo del Derecho, el Folklore y la Iconografía, la evaluación de resúmenes, la tasación de documentos, la historia de la catalogación, la integración educativa supranacional en la formación de profesionales de la información, la aplicación de metodologías activas y de formación integral y ecológica en la docencia, los archivos de las organizaciones empresariales y los medios de comunicación, y los estudios de usuarios en el campo de la Archivística. Se observa la transferencia de valores, programas y metodologías entre las distintas áreas de investigación; la importancia de las actividades de armonización profesional y desarrollo humano como motor de la investigación y la práctica profesional; y la apertura hacia formas de docencia más activas y ecológicas.

Palabras clave: Ciencia de la Información. Organización del conocimiento. Catalogación. Formación profesional. Archivística. Tendencias de investigación. Editorial. Revisión.

\subsection{Abstract}

Presentation and analysis of some challenges in Information Science research as represented by the articles published in Scire, volume 10, number 1 (JanuaryJune 2004). Those papers consider mainly new research topics in the fields of History of Documentation, the theoretic background of content analysis, indexing languages in the fields of Law, Folklore and Iconography, abstracts evaluation, document appraisal, history of cataloguing, regional educational integration, active and ecological teaching methodologies, business associations archives, media archives, and users' studies in the archival field. A transference of values, 
models and techniques among the different subdisciplines can be observed, and also the importance of internationalization as an engine for the development of research and practice, and the progressive importance of more active and ecological methodologies in formal education.

Keywords: Information Science. Knowledge Organization. Cataloguing. Professional education. Archival Science. Research trends. Editorial. Review.

\section{Introducción}

El presente número de Scire ofrece catorce estudios que se pueden agrupar en seis temas: historia e identidad de las Ciencias de la Documentación (Sagredo, 2004), análisis documental del contenido (Izquierdo Arroyo, 2004; Moreno y Caparrós, 2004; Tomás, 2004; Alvite, 2004; Moreno y del Baño, 2004) y formal (Pedraza, 2004; López, 2004), educación y formación (Oliveira y Guimarães, 2004; Vieira y Silva, 2004; Ferrer, Peset y Lloret, 2004), Archivística (del Prado, 2004; Rubio, 2004) y documentación de medios de comunicación (Martín y Sánchez, 2004). Son estudios diversos cuyo denominador común es, con alguna excepción, la exploración de temas y metodologías nuevas en nuestro contexto cultural. En este sentido, se pueden considerar representativos de algunos de los frentes de investigación que se van abriendo en las diferentes disciplinas documentales. A continuación, presentaremos en los consiguientes apartados las, a nuestro juicio, principales aportaciones de estos trabajos y exploraremos algunas sugerencias y perspectivas que se derivan de ellos.

\section{Las raíces universalistas de la Documentación}

La Biblioteconomía como disciplina ha poseído siempre una perspectiva y unas raíces que transcienden el ámbito global. Sus más grandes hitos están relacionados con proyectos internacionales, como la Biblioteca de Alejandría o la red informal - pero muy eficaz - que formaron las bibliotecas monásticas altomedievales. Felix Sagredo (2004) explora unas raíces más inmediatas que han tenido un gran impacto en la construcción de la Documentación como ciencia en nuestro país a través del núcleo de la Universidad Complutense de Madrid, a saber: el compromiso internacionalista de dos de los padres europeos de la Documentación - Paul Otlet y Henri Lafontaine - y su importancia en la configuración de la nueva ciencia. Tras analizar los principales hitos internacionalistas de Paul Otlet, y en particular, su papel pionero en la redacción de una carta de las Sociedad de Naciones, Sagredo (2004, p. 25) sintetiza el impacto de su compromiso político en la nueva disciplina:

Su misma concepción como tal es fruto de algo que se deriva directamente del positivismo filosófico del que la Documentación toma carta de naturaleza. Los primeros documentalistas no concibieron este nuevo campo del saber como un instrumento

Scire. $10: 1$ (en.-jun. 2004) 9-20. 
aséptico; sino como un catalizador de nuevas ideas que fueran compartidas por todos los humanos, especialmente los dedicados al cultivo del intelecto y de la información. Podríamos incluso afirmar, sin duda alguna, que tanto Henri La Fontaine como Paul Otlet concibieron la Documentación como un instrumento esencial de la Paz anhelada para la Europa contemporánea y para el mundo en general.

Aunque el compromiso social y político de muchas de las grandes figuras de las Ciencias de la Documentación es bien conocido, este trabajo demuestra como el proyecto científico de Paul Otlet y Henri Lafontaine era solo una parte de un proyecto mayor de carácter universalista y pacifista. La importancia de los movimientos internacionalistas en el avance de las disciplinas documentales se podrá observar también desde dos ópticas completamente distintas - la normalización catalográfica y la armonización curricular- en dos de los estudios que se analizarán a continuación (López Guillamón, 2004; Oliveira y Guimarães, 2004).

\section{Representación y organización documental}

El bloque más importante de estudios publicados en este número - siete trabajos - tiene que ver con lo que en el ámbito francófilo se denomina análisis documental y en el anglosajón catalogación. El primero de los artículos es un estudio de Mónica Alonso (2004) sobre las estructuras retóricas, útil especialmente para la fundamentación teórica del análisis de contenido y la confección de resúmenes; los tres siguientes se dedican a los lenguajes documentales en diversos ámbitos - la iconografía y el arte (Moreno y Caparrós, 2004), el folklore (Tomás, 2004) y el mundo jurídico (Alvite, 2004) -; el cuarto se ocupa de la evaluación de resúmenes (Moreno y del Baño, 204); el quinto de la tasación como operación documental (Pedraza, 2004); y el último de la historia de la normalización de la catalogación bibliográfica (López Guillamón, 2004).

Mónica Izquierdo Alonso (2004) sitúa como énfasis de su investigación sobre análisis documental del contenido (ADC) el estudio de los aspectos retóricoformales del texto - a los que denomina "Forma Documental del Contenido"-, ya que "el ADC, tanto desde su dimensión teórica como desde su formulación metodológica, se sigue centrando en la macroestrutura temática" (ibidem, p. 34). Desde nuestro punto de vista, una de las aportaciones más interesantes de su trabajo es el énfasis que realiza en el estudio de la relación entre las estructuras lingüísticas reales y los procedimientos y productos del análisis documental del contenido. Este enfoque es el único susceptible de conducir, al final, a una representación formal de esos procesos, que es la que va a permitir su homogeneización, su evaluación y su eventual automatización más allá de la aplicación de criterios parciales o subjetivos basados en el consenso de expertos. Este último es un procedimiento válido para el avance práctico de las disciplinas, pero la fundamentación científica requiere avanzar en la medida de lo posible en una explicación sistemática y determinista, al menos como objetivo. Además, hay que Scire. $10: 1$ (en.-jun. 2004) 9-20. 
destacar el trabajo empírico de análisis que la autora ha realizado sobre un corpus de artículos científicos, que es el género escogido como ejemplo para su estudio aplicado. En conclusión, el estudio de las estructuras retóricas es el puente entre el micro-análisis terminológico y el macro-análisis textual, y con ellos uno de los pilares que sostienen el desarrollo científico del análisis de contenido.

El artículo de Moreno y Caparrós (2004) está dedicado a presentar con detalle en español la clasificación iconográfica Iconclass. Los autores analizan su utilización en bases de datos iconográficas, su estructura y funcionamiento, y la comparan con los lenguajes de indización más utilizados. Además de por su extraordinaria factura y calidad, el interés de Iconclass se acrecienta en la medida en que es un lenguaje pionero en enfrentarse al medio contemporáneo por excelencia: el visual y multimedia. Lo icónico es uno de los elementos clave, aunque no el único, del medio visual, y constituye respecto a la imagen lo que el concepto de tema es al texto. Los lenguajes documentales que se ocupan de la "imagen culta" sirven también de comparación y de perspectiva complementaria al otro gran desarrollo de la organización del conocimiento en el mundo visual: los tesauros de imagen especialmente desarrollados para los medios de comunicación - prensa y televisión, fundamentalmente - . Interesa aquí resaltar como las necesidades del uso científico, que exigen una recuperación muy precisa y exhaustiva, han encontrado una respuesta más adecuada en las clasificaciones, que aseguran la asignación sistemática de los documentos a una estructura conceptual con capacidades predictivas. Por el contrario, los lenguajes de los medios de comunicación, que más bien están orientados a buscar ejemplares representativos, se han conformado con tesauros. Surge aquí un paralelismo importante entre los usos de los lenguajes documentales y su forma: cuando el lenguaje documental busca ordenar el campo primario de estudio, la clasificación es una necesidad ineludible - de la misma forma que ocurre con las taxonomías en las Ciencias Naturales - para revelar la estructura interna de un campo y organizar el conocimiento de forma sistemática. Sin embargo, cuando el lenguaje documental busca sobre todo recuperar para ilustrar o resolver una necesidad de información genérica caben otras aproximaciones menos sistemáticas.

A continuación, Miriam Tomás (2004) presenta los resultados de su investigación doctoral sobre el diseño y realización de un tesauro de folklore y oficios artesanales de la región de Murcia. Este trabajo aporta, en primer lugar, el propio tesauro con sus ocho áreas - vestuario, instrumentos musicales, cancionero, comarcas de la Región de Murcia, juegos infantiles, intérpretes, manifestaciones de folklore y oficios populares - . Además, presenta innovaciones metodológicas, en particular, la utilización de técnicas etnográficas para la recuperación y definición del vocabulario, a partir de entrevistas semiestructuradas de carácter abierto con personas cualificadas: (ibidem, p. 75):

Scire. $10: 1$ (en.-jun. 2004) 9-20. 
En el Tesauro Conceptual de Folklore de la Región de Murcia, la presencia del usuario ha sido de vital importancia y pieza fundamental y decisiva para la construcción del mismo. La aplicación de las técnicas de investigación cualitativa en Biblioteconomía y Documentación no sólo ayuda a estudiar el comportamiento de los usuarios en la fase de búsqueda y recuperación de información; sino que, además permite extraer de ellos los diferentes puntos de vista y las estructuras conceptuales del campo de estudio en cuestión.

En la misma línea de investigaciones aplicadas a un campo específico del saber, Alvite ofrece un compleo estudio de los instrumentos terminológicos integrados en las interfaces de las bases de datos jurídicas Aranzadi, Iberlex y La Ley, las más utilizadas en España. El ámbito jurídico destaca por haber sido uno de los pioneros a nivel internacional en el desarrollo de bases de datos a texto completo. El artículo parte de la consideración de las necesidades de información de los usuarios jurídicos, de las que deriva los requerimientos de los lenguajes documentales necesarios y el patrón a seguir para la evaluación de los actuales. Como en otros campos profesionales especializados, las consultas de los juristas son básicamente de dos tipos: a) búsquedas muy precisas sobre "campos factuales" - fechas de emisión, de publicación, número de la disposición, etc.-—; y b) búsquedas temáticas, para las cuales resulta necesario superar la aproximación tradicional basada en índices analíticos de términos y apoyarse en sistemas que, como los tesauros, representan los conceptos y su relaciones de forma precisa, y que, con el tiempo, hagan posible una indización asistida por ordenador.

El trabajo de Moreno y del Baño (2004) aborda la evaluación de otra de las grandes herramientas de recuperación: los resúmenes. En particular, estudia el grado de cumplimiento de las recomendaciones y normas prescritas por las revistas científicas sobre resúmenes de autor a partir del estudio de los artículos del año 2000 de las revistas Fisioterapia y la Revista Española de Documentación Científica. El modelo parte de las recomendaciones propuestas por la norma UNE 50-103-90 y también de las específicas propuestas por los propios editores de las revistas; y estudia el tipo, presentación y estilo de los resúmenes, y la presencia o no de palabras clave o descriptores libres. Los resultados sirven para conocer los errores más frecuentes cometidos por los autores: la presencia de mucha información secundaria, la falta de claridad y precisión, y la falta de correspondencia entre la extensión y estructura de los resúmenes y el contenido sustancial de los artículos. Aunque la utilidad del resumen pasa muchas veces desapercibida entre las personas que desarrollan su formación y su labor profesional lejos de los ámbitos de la investigación, su importancia es grande en la documentación científica, y también, como señalan los autores, para la promoción de la investigación a través de las bases de datos internacionales que permiten su difusión y sirven de instrumento para la evaluación científica. Este artículo revindica indirectamente su importancia y utilidad.

Scire. $10: 1$ (en.-jun. 2004) 9-20. 
En el artículo siguiente, Manuel Pedraza (2005) intenta una ampliación del modelo clásico de procesamiento documental - la, en nuestro ámbito, popular "cadena documental" - para incluir una función crítica en las bibliotecas nacionales y regionales, las librerías anticuarias y otros entornos que trabajan con el libro antiguo: la tasación. Su objetivo específico es establecer el proceso de tasación como proceso de tratamiento documental y fijar las relaciones que se producen entre el libro como documento primario y el informe de tasación o el catálogo como documento secundario. Para el autor (ibidem, p. 119), la tasación añade elementos específicos al análisis formal y de contenido, pero básicamente se inserta con ellos en el mismo proceso de información documental:

La tasación del libro es un proceso en el que la información constitutiva de un documento primario - un ejemplar - se expresa de forma sintética mediante un conjunto de procedimientos técnicos especializados de carácter analítico en otro documento de carácter secundario - tasación- cuyo fin es proporcionar una valoración - no sólo económica - del documento objeto de estudio. Como es conocido, estos procedimientos analítico-sintéticos se denominan en documentación tratamiento documental.

Finalmente, cerrando este conjunto de estudios sobre análisis documental, López Guillamón (2004) presenta un extenso estudio cuyo objetivo es realizar una revisión bibliográfico-histórica de la catalogación en los dos últimos siglos. El autor muestra cómo se formó el concepto de catalogación internacional a partir de, en primer lugar, la idea de cooperación entre bibliotecas y, en segundo lugar, del esfuerzo de normalización de los procedimientos de catalogación. En la actualidad, la automatización se ha convertido en otra de las fuerzas que apoyan el proceso. López Guillamón resume así esa historia (ibidem, p. 140):

En los inicios los principios de normalización bibliográfica y de unificación reglamentaria actuaron como estímulos para alcanzar la cooperación bibliotecaria en materia de catalogación por razones pragmáticas. Los principios de la catalogación de uso común, precisión y significación fueron los móviles que han llevado más recientemente a las reglamentaciones catalográficas a disponer de una articulación cohesionada, que ha permitido el libre intercambio de los registros bibliográficos. Este proceso revierte en la universalización de la teoría de la catalogación, sometida en el presente, y en el futuro próximo, a contrastar las tradiciones prácticas, bajo el patrocinio de la IFLA y de las principales instituciones bibliotecarias.

\section{La educación y formación de los profesionales de la información: la búsqueda de un aprendizaje relevante, activo, significativo, aplicado y sistemático}

El siguiente bloque de estudios de este número lo representa el de artículos dedicados a la formación y educación de los profesionales actuales y futuros de la información y la documentación. El primer artículo está dedicado a la presentación del proceso de integración curricular que se está produciendo en el Mercosur,

Scire. $10: 1$ (en.-jun. 2004) 9-20. 
y ofrece su experiencia en la definición de las áreas de estudio troncales (Tannuri y Guimarães, 2004). Los dos siguientes presentan sendas experiencias docentes innovadoras en la aplicación de los mapas conceptuales a la docencia (Vieira y Silva, 2004) y la integración interdisciplinar de equipos de estudiantes de distintas asignaturas y titulaciones (Peset, Ferrer y Lloret, 2004).

El trabajo de Tannuri y Guimarães (2004) estudia la evolución de la formación superior del profesional de la información en el Mercosur, como especial atención al proceso de armonización curricular que se inicia a partir de 1996. Se relatan las aportaciones de los Encuentros de Directores y Docentes de los Cursos Superiores de Bibliotecología del Mercosur, una experiencia modélica de integración regional que puede servir de punto de comparación a otras iniciativas como la que actualmente se está produciendo en Europa con el Espacio Europeo de Educación Superior. El punto actual y la filosofía del proceso, según la señalan los autores (ibidem, p. 153) tiene, sin duda, validez general para la integración regional en nuestras disciplinas:

Cada uno de los países del Mercosur se encuentra en un momento diferente en lo que concierne al desarrollo y a la implementación de políticas para la enseñanza superior, hecho éste que se refleja también en la enseñanza de la Biblioteconomía. Una posible integración requiere minimizar estos puntos de diferencia, no en el sentido de nivelar las características, sino en el sentido de encontrar puntos de identidad entre los diferentes sistemas de educación superior, tanto en lo que concierne a la organización y objetivos de los cursos como en lo relacionado a la propia estructuración curricular.

Dos de las conclusiones más importantes obtenidas en los citados Encuentros también son de gran interés para el resto de los países: la inserción de la investigación como pilar transversal de la formación de los profesionales de la información, y la importancia de la formación permanente. Respecto a la investigación, los autores se expresan así: “[...] aumentar la investigación en la graduación es una propuesta que ofrece un elemento de intervención en lo cotidiano, garantizando un egresado apto para desafiar circunstancias diversas en el mercado de trabajo, capaz de actuar en diversas instancias y en un universo geográfico más amplio."

En la línea de los pedagogos constructivistas, especialmente de Ausubel y Novak e inspirándose en la filosofía de la educación de Morin, Vieira y Silva (2004) se plantean la necesidad de que la formación profesional del profesional de la información dote a los estudiantes de herramientas para toda la vida, para aprender a aprender. Esta es la filosofía que les llevó a integrar la enseñanza con mapas conceptuales en su labor como profesoras del Curso de Biblioteconomía de la Universidade Federal de Santa Catarina (Florianópolis, Brasil). Más allá de su utilidad obvia para la enseñanza del análisis de contenido y los lenguajes documentales - cuyo interés para la enseñanza del análisis de contenido también han analizado otros autores (García, 1997) —, las autoras resaltan la utilidad

Scire. $10: 1$ (en.-jun. 2004) 9-20. 
de la herramienta para compartir en clase las distintas "redes de significados", estimulando el pensamiento reflexivo y la construcción del conocimiento, tanto individual como compartida. Vieira y Silva muestran como el uso de los mapas conceptuales es eficaz para despertar en los estudiantes la capacidad de situar la información en su contexto e integrarla en la realidad; y como resultan útiles como una metodología docente muy valorada por los estudiantes de la enseñanza superior para la asimilación de asignaturas de calado teórico (ibidem, 167):

En este tipo de aprendizaje, el profesor no es un trasmisor de conocimientos, sino un orientador; el instigador de un proceso que debe ser interactivo y permanente. A través del aprendizaje significativo el estudiante tiene un compromiso y una responsabilidad con el proceso de aprender. Esta forma de aprendizaje ocurre cuando el estudiante establece relaciones entre los nuevos conceptos o las nuevas informaciones y los conceptos y conocimientos que ya posee. Este aprendizaje se contrapone al "aprendizaje memorístico donde la información nueva no se asocia con los conceptos existentes en la estructura cognitiva, donde se produce una interacción mínima o nula entre la información recientemente adquirida y la información ya almacenada." (Ontoria Peña, 1996, p. 27). Los mapas conceptuales son instrumentos pedagógicos que auxilian en la formación de una "cabeza bien hecha", porque permiten que los que los estudiantes, en vez de dedicarse a una acumulación pura y simple del saber, pasen a disponer de una "aptitud general para colocar y tratar problemas y principios organizadores que permiten unir los saberes y darles sentido" (Morin, 2000, p. 21). La educación, en el siglo XXI, debe ser un proceso para toda la vida. Debe preocuparse por la formación del ciudadano y de la persona y no solo de su formación profesional. Aprender a aprender es un jalón fundamental de ese camino.

Finalmente, Ferrer, Peset y Lloret (2004) presentan un proyecto piloto de innovación docente pluridisciplinar realizado en la Universidad Politécnica de Valencia en el marco del proceso de ajuste al Espacio Europeo de Educación Superior. La experiencia consistió en integrar las actividades de formación de estudiantes de Documentación y Bellas Artes en torno a la realización de documentales sobre artistas, instituciones u organizaciones relacionadas con el Arte Contemporáneo valenciano, con el fin de emitirlos por la Televisión de la Universidad Politécnica. La principal aportación de estas interesantísimas metodologías de proyectos de alta validez "ecológica" - en el sentido de que procuran el aprendizaje en entornos educativos cercanos al futuro campo de desarrollo profesional - es su cercanía a la realidad del mercado laboral. Efectivamente. en el mundo laboral el trabajo es individual, pero también en equipo; exige especialización, pero también capacidad para interaccionar en grupos de profesionales con perfiles heterogéneos. En él es necesario atender al entorno real; procurar la consecución de objetivos específicos, relevantes y mensurables; cumplir rigurosamente los plazos; $y$ someterse a unos usuarios o clientes que van a evaluar inmediatamente el resultado de su trabajo. Todo esto se consigue con la metodología presentada por las autoras, que permite que los alumnos interioricen de verdad los

Scire. $10: 1$ (en.-jun. 2004) 9-20. 
contenidos, aprendidos muchas veces tan solo en teoría y de forma memorística. Cabe destacar, por último, la exigencia de un informe final del proyecto, que en este tipo de experiencias educativas permite al grupo de estudiantes reflexionar, verbalizar y conectar con los modelos y el vocabulario de su disciplina una experiencia práctica, que, por otra parte, muchas veces pierde fuerza educativa cuando se queda tan solo en la sola ejecución de tareas y realización de productos.

\section{La Archivística: una disciplina en expansión}

Por fin, cierran el número un conjunto de estudios sobre archivística aplicada, que abordan temas menos usuales dentro de la corriente general de la disciplina, al menos en el mundo hispanoamericano.

El primero de ellos es una exhaustiva investigación por Miguel Ángel del Prado (2004) sobre la situación de los archivos de las organizaciones empresariales en España, que puede servir de modelo para el abordaje de otros conjuntos de archivos poco estudiados. El trabajo parte del análisis de la historia del asociacionismo empresarial español desde finales del siglo XVIII hasta la nuestros tiempos, deslindando la evolución institucional de estos organismos y la relación entre ellos. Posteriormente, se examinan las distintas fuentes de información existentes sobre estos archivos y sus fondos documentales, presentando un mapa prospectivo. Seguidamente, se presenta el estado de la cuestión de la Archivística en el campo de las organizaciones empresariales y el marco legal que se aplica a sus documentos, con especial atención a sus características distintivas. Se llega así a un diagnóstico de sus principales problemas: falta de profesionales, vacío legal en materia documental, ausencia de información y de bibliografía específica, y peligro de desaparición de la documentación con valor histórico. A partir del diagnóstico se plantea un conjunto de propuestas de actuación realistas que parten de la consideración del archivo como una pieza fundamental del sistema organizativo y de funcionamiento interno de las organizaciones, y de la necesidad de una visión global que abarque el ciclo vital completo de la documentación y los diferentes soportes documentales. Las dos propuestas más notables son la integración del archivo, la biblioteca y el centro de documentación en un sistema de gestión documental con un técnico polivalente a su cargo, y, a medio plazo, el establecimiento de fundaciones para hacerse cargo de forma cooperativa del mantenimiento de este patrimonio documental histórico. Las nuevas tecnologías ocupan un lugar fundamental, como no podría ser de otro modo, en este proyecto de facilitar la conservación y puesta en servicio del patrimonio documental de esta organizaciones, articuladas en torno al concepto de archivo virtuale.

El estudio de Alfonso Rubio (2004) aborda el estudio de usuarios en el campo de los archivos, un área a la que se suele prestar menos atención frente a la conservación y el acceso. Su aportación tiene también un indudable valor

Scire. $10: 1$ (en.-jun. 2004) 9-20. 
metodológico, a la vez que se apoya en un sólido estudio de caso realizado en el Archivo Municipal de Logroño (Comunidad Autónoma de La Rioja, España). En primer lugar, se establecen las fuentes a partir de las cuales realizar la investigación. En el presente estudio son tres fuentes de carácter indirecto: el Libro registro de préstamos y consultas de usuarios internos y el Libro registro de ciudadanos y Fichero de investigadores. En segundo lugar, se establece la tipología de usuarios a estudiar, que el autor divide, como es frecuente, en internos - gestores administrativos - y externos - investigadores y ciudadanos - . Seguidamente, se describen sus características, se analizan los resultados y se realiza un diagnóstico de la situación real. Finalmente, se presentan propuestas de mejora del funcionamiento en relación a cada tipo de usuario y a la información que suelen recabar.

Finalmente, el estudio de Yolanda Martín y María F. Sánchez (2004) es una breve comunicación que sintetiza el estado de los archivos audiovisuales y de los centros de documentación en algunos de los principales periódicos nacionales por contraposición al ámbito local. En concreto la muestra está compuesta, en el ámbito nacional, por los diarios El País, $A B C$ y El Mundo, y, en el local, por dos periódicos de la provincia de Salamanca, La Gaceta Regional y El Adelanto. Entre los resultados cabe destacar el enorme y creciente impacto de la Internet en la difusión de la prensa y en el trabajo de documentación y archivo; la consolidación de la hemeroteca digital como un producto más de los rotativos gracias a la tecnología digital, y la lenta pero imparable penetración de especialistas en documentación en el ámbito de los medios de comunicación, mucho mayor, como ya se ha señalado en esta revista anteriormente, en los medios nacionales que en los locales.

\section{Conclusión}

El análisis de los trabajos publicados en el presente número permite sacar algunas conclusiones de interés sobre algunos problemas candentes en nuestra disciplina y sobre sus requisitos de desarrollo científico y social.

En primer lugar, los estudios de Sagredo (2004), López Guillamón (2004) y Oliveira y Guimarães (2004) muestran como la Ciencia de la Documentación se apoya como otras ciencias en valores como la cooperación internacional entre los investigadores y especialistas. Precisamente en este valor encuentra uno de sus referentes más importantes. Trabajar en pro de la comunicación sin fronteras del conocimiento es uno de los principales motores de la teoría y la práctica de la Documentación, pero también una de las condiciones necesarias de un trabajo fructífero en el medio y largo plazo.

En segundo lugar, es necesario resaltar la importancia de una buena transferencia de ideas, modelos y técnicas entre las Ciencias de la Documentación y las disciplinas en las que éstas se apoyan $-\mathrm{y}$ que son muy numerosas y variadas,

Scire. $10: 1$ (en.-jun. 2004) 9-20. 
desde la Lingüística (Izquierdo, 2004) hasta la Administración, pasando por la Informática - y también entre sus propias subdisciplinas, como ejemplifica el estudio publicado en este número sobre la aplicación de los estudios de usuarios a la Archivística (Rubio, 2004). Esto supone, recíprocamente, ampliar modelos generales - como el del proceso o cadena documental - para incorporar casos específicos, como sucede en la propuesta que realiza Pedraza (2004) de incorporar la tasación como operación al modelo clásico de procesamiento documental.

En tercer lugar, es necesario enfatizar la importancia de extender la investigación aplicada de carácter informativo-documental a todos los campos del saber y de la actividad humana. En este número, se presentan trabajos en campos muy atendidos por las Ciencias de la Documentación en España, como es el caso de la Documentación de Medios de Comunicación (Martín y Sánchez, 2004), pero también en otros más descuidados como la Documentación Jurídica (Alvite, 2004) o el Folklore (Tomás, 2004).

Por fin, se debe destacar la importancia de realizar una transferencia interna de conocimientos - esa es la función de la docencia en cualquier disciplina, al fin y al cabo - que se base en un diagnóstico preciso de al menos cuatro factores clave: los objetivos de formación, el estado de los estudiantes, las características del contexto de la interacción educativa y el estado de la teoría y la práctica pedagógica. En este sentido, es necesario resaltar la necesidad de completar la formación técnica con la adquisición y desarrollo de competencias metacognitivas (Cunha y Silva, 2004) y relacionales (Peset, Ferrer y Looret, 2004) de carácter general, que muchas veces se dan por supuestas, y que son fundamentales para el éxito profesional y personal de los estudiantes. También conviene enfatizar la necesidad de que los métodos docentes sean coherentes con los objetivos que se pretenden conseguir. En este sentido, los primeros cursos deberían orientarse a la consecución de conocimientos y habilidades de modo analítico - con el objetivo de conseguir conceptos y "automatismos" -, mientras que en los últimos cursos se deberían proponer actividades docentes orientadas a la integración de habilidades en entornos "semejantes" a los que el egresado va a encontrar en el futuro.

\section{Referencias}

Alvite Díez, María Luisa (2004). Las herramientas terminológicas en los sistemas de información jurídica. // Scire: representación y organización del conocimiento. 10: 1 (en.-jun. 2004) 77-90.

Cunha, Miriam Vieira da; Silva, Edna Lúcia da (2004). Redes semánticas y mapas conceptuales: una experiencia en la formación de bibliotecarios. // Scire: representación y organización del conocimiento. 10:1 (en.-jun. 2004) 159-168.

García Marco, Francisco Javier (1997). Implicaciones teóricas y didácicas de la técnica de los mapas conceptuales para la enseñanza del análisis de contenido y los

Scire. $10: 1$ (en.-jun. 2004) 9-20. 


\section{Francisco Javier García Marco}

lenguajes documentales. // García Marco, Francisco Javier (editor). Organización del Conocimiento en Sistemas de Información y Documentación: 2. Zaragoza: Universidad, 1997. 175-186.

Izquierdo Alonso, Mónica(2004). Nuevos retos en el análisis documental de contenido: la gestión de la forma documental del contenido. // Scire: representación y organización del conocimiento. 10:1 (en.-jun. 2004) 31-50.

López Guillamón, Ignacio (2004). Apuntes para una historia de la catalogación internacional en los siglos XIX y XX. // Scire: representación y organización del conocimiento. 10:1 (en.-jun. 2004) 121-144.

Martín González, Yolanda; Sánchez Hernández, María F. (2004). La actividad documental en el ámbito periodístico: análisis comparativo y perspectivas. // Scire: representación y organización del conocimiento. 10:1 (en.-jun. 2004) 237-241.

Moreno Fernández, Luis Miguel (2004). Iconclass: Clasificación Iconográfica para la ordenación, resumen e indización de la documentación icónica. // Scire: representación y organización del conocimiento. 10:1 (en.-jun. 2004) 51-64.

Moreno Fernández, Luis Miguel ; Baño Aledo, María Elena del (2004). La calidad del resumen en las publicaciones científicas españolas: estudio de caso. // Scire: representación y organización del conocimiento. 10:1 (en.-jun. 2004) 91-100.

Morin, E. (2000). A cabeça bem-feita: repensar a reforma, reformar o pensamento. Rio de Janeiro: Bertrand Brasil, 2000.

Oliveira, Ely Francina Tannuri de; Guimarães, José Augusto Chaves (2004). Políticas de formación profesional en el área de información en el Mercosur: reflexiones acerca de una experiencia de armonización curricular en Biblioteconomía. // Scire: representación y organización del conocimiento. 10:1 (en.-jun. 2004) 145-157.

Ontoria Peña, A. et al. (1996). Los mapas conceptuales en el aula. Buenos Aires: Ed. Magisterio del Rio de La Plata, 1996.

Pedraza Gracia, Manuel José (2004). Los procesos informativo-documentales de la tasación del libro antiguo. // Scire: representación y organización del conocimiento. 10:1 (en.-jun. 2004) 101-120.

Peset Mancebo, Fernanda; Ferrer Sapena, Antonia; Lloret Romero, Nuria (2004). El aprendizaje desde la práctica: integración de nuevas metodologías docentes en Documentación. // Scire: representación y organización del conocimiento. 10:1 (en.jun. 2004) 169-183.

Prado Martínez, Miguel Ángel del (2004). La Archivística en el ámbito de las organizaciones empresariales: estado de la cuestión y propuestas de actuación. // Scire: representación y organización del conocimiento. 10:1 (en.-jun. 2004) 185-216.

Rubio Hernández, Alfonso (2004). Estudios de usuarios en archivos municipales: una aproximación teórico-práctica. // Scire: representación y organización del conocimiento. 10:1 (en.-jun. 2004) 217-236.

Sagredo Fernández, Félix (2004). La Documentacion y el nacimiento de las Naciones Unidas. // Scire: representación y organización del conocimiento. 10:1 (en.-jun. 2004) 19.

Tomás López, Miriam (2004). Diseño de un tesauro conceptual de folklore de la Región de Murcia. // Scire: representación y organización del conocimiento. 10:1 (en.-jun. 2004) 59.

Scire. $10: 1$ (en.-jun. 2004) 9-20. 